\title{
Older adults have difficulty in decoding sarcasm
}

\author{
Second revision submitted to Developmental Psychology $11^{\text {th }}$ August 2015
}

Author information:

Louise H. Phillips, Roy Allen \& Rebecca Bull: School of Psychology, University of Aberdeen; Alexandra Hering \& Matthias Kliegel: Department of Psychology, University of Geneva; Shelley Channon, Department of Experimental Psychology, University College London.

Roy Allen died during the course of preparing this manuscript.

Rebecca Bull is now at the National Institute for Education, Singapore.

This research was funded by the Leverhulme Trust, U.K. (F/00152/W). We would like to acknowledge the assistance of Francis Quinn in collecting the data.

Correspondence should be addressed to Louise H. Phillips, School of Psychology, College of Life Sciences and Medicine, University of Aberdeen, Aberdeen, Scotland, UK, AB24 2UB. Tel (+44) 1224 272229. Email: louise.phillips@abdn.ac.uk. 


\section{Older adults have difficulty in decoding sarcasm.}

Younger and older adults differ in performance on a range of social cognitive skills, with older adults having difficulties in decoding nonverbal cues to emotion and intentions. Such skills are likely to be important when deciding whether someone is being sarcastic. In the current study we investigated in a lifespan sample whether there are age-related differences in the interpretation of sarcastic statements. Using both video and verbal materials, 116 participants aged between 18 and 86 completed judgments about whether statements should be interpreted literally or sarcastically. For the verbal stories task, older adults were poorer at understanding sarcastic intent compared to younger and middle-aged participants, but there was no age difference in interpreting control stories. For the video task, older adults showed poorer understanding of sarcastic exchanges compared to younger and middle-aged counterparts, but there was no age difference in understanding the meaning of sincere interactions. For the videos task, the age differences were mediated by the ability to perceive facial expressions of emotion. Age effects could not be explained in terms of variance in working memory. These results indicate that increased age is associated with specific difficulties in using nonverbal and contextual cues to understand sarcastic intent.

Keywords: LIFESPAN DEVELOPMENT, AGING, EMOTION PERCEPTION, THEORY OF MIND, SOCIAL DECODING.

Word count: 8897 
In contrast to early reports of preserved or improved ability to decode social cues across the lifespan (e.g. Happé, Winner, \& Brownell, 1998), older adults have difficulty with many aspects of social cue decoding (for a review see Phillips, Slessor, Bailey \& Henry, 2014). For example, there are agerelated declines in perceiving emotional cues from faces, voices and body movements (Mill, Allik, Realo \& Valk, 2009; Ruffman, Henry, Livingstone \& Phillips, 2008), indicating that interpersonal affect is more difficult to decode in old age. Also, there are age-related declines in understanding the beliefs and intentions of other people, as conveyed by their speech, gestures or facial expression (for a metaanalysis of age differences in these different aspects of 'theory of mind' see Henry, Phillips, Ruffman \& Bailey, 2013).

In everyday interaction, people draw conclusions about the intentions and emotions of others based on a range of cues such as facial expression, bodily movement and the tone or content of speech. Sometimes the only way to accurately interpret someone's mental state is to go beyond the literal meaning of what they say, and use paralinguistic and contextual cues to decipher their true intention. Literal meaning and true intentions may conflict when a speaker is using humor, being sarcastic, or attempting to deceive. Research indicates that older adults sometimes have difficulty in understanding non-literal statements. For example, there are age differences in appreciation of some types of humor (Uekermann, Channon \& Daum, 2006) and interpreting cues to deception (Ruffman, Murray, Halberstadt \& Vater, 2012; Stanley \& Blanchard-Fields, 2008). Older adults have also been reported to have problems in interpreting non-literal statements such as proverbs or metaphors (e.g. Uekermann, Thoma \& Daum, 2008). However, some evidence indicates that there may be no age differences in interpreting metaphors (Champagne, Jean-Louis \& Joannette, 2006), or indeed age-related improvements in detecting lies (Bond, Thompson \& Malloy, 2005).

A common type of non-literal statement occurs when a speaker is using sarcasm. Sarcasm is present when remarks are made with an underlying negative intent but there is a discrepancy with the 
literal meaning or context (e.g. Dews et al., 1996). For example, if a colleague habitually arrives late for meetings and turns up late once again, someone waiting might look at their watch and say 'Good to see you are on time as usual'. The literal meaning of sarcastic remarks is often positive, but context, tone of voice and exaggerated gestures often convey that the intended meaning is in fact the opposite. Understanding the intention of a speaker who is being sarcastic requires the ability to interpret pragmatic cues (i.e. those cues which come from the social context), and suppress the literal meaning of what is said. To date there has been little research investigating the effects of normal aging on the ability to interpret cues to sarcasm, and this issue is addressed in the current research.

It is important to note that sarcasm is not intended to be deceptive - the speaker uses the conflict between context and meaning or the exaggerated paralinguistic cues to flag to the listener that they are being sarcastic. The same types of facial and bodily cues which are important in interpreting emotions are likely to be important in detecting sarcasm. More complex theory of mind skills involved in understanding mental states are also expected to be important in decoding the meaning of non-literal statements (Shamay-Tsoory, Tomer, \& Aharon-Peretz, 2005). For example, in order to detect that the meaning of a statement is sarcastic rather than literal, it may be necessary to understand the knowledge of the people involved in the interaction, and the intention of the person making the statement.

Older adults perform poorly on many theory of mind tasks (Henry et al., 2013) and this has been linked to lower integrity of white matter pathways in the brain (Charlton, Barrick, Markus \& Morris, 2009). Parallels have been drawn (e.g. Ruffman et al., 2008; Slessor, Phillips \& Bull, 2008) between older adults' performance on social decoding tasks and that shown by other populations whose corticalsubcortical white matter connectivity is compromised. The ability to decode sarcasm is impaired following frontal lobe brain injury (Channon et al., 2007), and in frontotemporal dementia (Kipps, Nestor, Acosta-Cabronero, Arnold \& Hodges, 2009). Given age-related reductions in integrity of frontal 
white matter connectivity (e.g. Charlton et al., 2009) it can therefore be predicted that older adults may show problems in interpreting sarcastic statements.

If there are age-related changes in the comprehension of sarcasm, it is of interest to explore whether this relates to emotion perception and theory of mind. The ability to detect and interpret sarcasm has been found to relate to emotion recognition in people with brain injury (Shamay-Tsoory et al., 2005), and dementia (Kipps et al., 2009; Rankin et al., 2009; Shanay-Ur et al., 2011). There is also evidence that theory of mind abilities are related to sarcasm comprehension in brain injured samples (e.g. McDonald \& Flanagan, 2004; Channon, Pellijeff \& Rule, 2005; Shamay-Tsoory et al., 2005; Winner, Brownell, Happé, Blum \& Pincus, 1998). Given that there are well-established age-related declines in understanding expressions of emotion (Ruffman et al., 2008) and theory of mind skills (Henry et al., 2013) it is of interest to explore whether any age differences in sarcasm comprehension are related to these other key social cognitive skills. Indeed, there is evidence that age-related problems in understanding social appropriateness and deception may be partially mediated by difficulties with emotion perception (Halberstadt, Ruffman, Murray, Taumoepeau, \& Ryan, 2011; Ruffman, et al., 2012; Stanley \& Blanchard-Fields, 2008).

Another important factor in any age effects on sarcasm comprehension might be cognitive changes. Dual task studies indicate that decoding protagonists' emotions and intentions places demands on working memory (Phillips, Channon, Tunstall, Hedenstrom \& Lyons 2008; Bull, Phillips \& Conway, 2008). To understand sarcasm, others' intentions and emotions must be understood, maintained and updated, while also placing statements in the context of the on-going situation and weighing up possible literal and non-literal interpretations of meaning. It therefore seems plausible that working memory updating is important in sarcasm comprehension, and might be associated with any age differences found. One way in which we explore this issue in the current study is through the use of matched control conditions for the sarcasm tasks which have similar perceptual and cognitive load but do not 
require the understanding of non-literal statements. A further way of addressing this issue is by taking a separate measurement of working memory updating to explore its relationship to age differences in sarcasm understanding.

As well as factors such as problems with emotion perception or working memory updating, there might be other reasons that older and younger people differ in their interpretation of sarcasm. Sarcastic statements usually contain some negative intent, and older adults are known to show positivity biases in their interpretation of social information (for a review see Reed, Chan \& Mikels, 2014). For example older adults are more likely to disengage from negative personal comments (Charles \& Carstensen, 2008), and to have positive responses to a negative social situation (Luong \& Charles, 2014). It is possible, therefore, that positivity biases in old age might results in older adults being less likely to attribute sarcastic intent to another.

Aging may also differentially relate to the interpretation of different types of sarcasm. There are a number of ways in which sarcastic intent can be communicated. For example, sarcasm can be conveyed by the tone of voice and other paralinguistic cues such as facial expressions and exaggerated gestures (Rankin et al., 2009). McDonald, Flanagan and Rollins (2002) refer to this as simple sarcasm. To decode simple sarcasm it is necessary to pick up the relevant paralinguistic cues. In contrast, paradoxical sarcasm occurs when a statement can only make sense when interpreted non-literally. For example, in one scenario in McDonald et al.'s stimuli, a character says, "Are you sure you've got your passport?", to which another responds, "Oh, yes, I tore it up and threw it away". Here the meaning of the interchange is nonsensical unless interpreted as sarcastic. As the simple sarcasm scenarios involve interpretation of paralinguistic cues while paradoxical sarcasm scenarios do not, it can be argued that the simple sarcasm items could be more sensitive to problems with nonverbal cue decoding.

Direct sarcasm can also be distinguished from indirect sarcasm (Bucciarelli, Colle, \& Bara, 2003). Direct sarcasm is where the sarcastic statement is a reversal of the actual meaning, for example (from 
Channon et al., 2005): after seeing a terrible play someone says, 'That was a fantastic play you took me to see'. Indirect sarcasm is more subtle and does not convey the exact opposite of the literal meaning, for example: after beating a friend at tennis someone says, 'I suppose you'll say there is a hole in your racquet?' The literal meaning is not intended, but in this case the sarcasm is more nuanced. The understanding of direct sarcasm develops earlier in childhood than indirect sarcasm (Dews et al., 1996), suggesting that interpreting indirect sarcastic statements requires more mature cognitive and/or social skills. However Channon et al. (2007) found that people with frontal lobe injuries were equally impaired in decoding direct and indirect sarcastic statements.

In the current study we looked at the effects of lifespan aging on the comprehension of sarcasm in both stories and videos. Sarcasm can be conveyed in text through the mismatch between social context and literal speech meaning. The comprehension of sarcasm from stories is likely to be dependent on understanding this mismatch. In videos, a range of contextual and paralinguistic cues to intent (such as body language, tone of voice, facial expression) can be used to portray sarcasm. It was of interest to use both modalities as there is some evidence that age effects on other social decoding skills in standard theory of mind tasks can vary depending on the modality of presentation (Slessor, Phillips \& Bull, 2007). Also, using two different modalities allows us to understand whether the pattern of age effects seen generalizes across tasks which differ considerably in perceptual and attentional demands.

\section{Predictions and hypotheses}

Understanding sarcastic statements requires decoding of emotional cues and underlying intentions in order to make non-literal interpretations of speech. As older adults have been shown to have problems with emotion perception (Ruffman et al., 2008), understanding intentions (Henry et al., 2013) and non-literal interpretations (Uekermann et al., 2008), we predicted that older adults would have difficulties in sarcasm comprehension. Given previous evidence of specific age-related declines on social cognitive skills compared to non-social control tasks (e.g. Phillips et al., 2011; Sullivan \& Ruffman, 
2004), we predicted that older adults would be impaired in comprehending sarcasm to a greater extent than control tasks matched for cognitive demands but not involving sarcastic intent. We also explored the effects of age across the lifespan. Because most studies of age effects on social decoding use an extreme age groups design, there is relatively little evidence to evaluate whether social cognitive skills continue to mature into middle age, or instead begin to decline early in adulthood. Perception of basic emotions such as anger and sadness may show age-related declines quite early in adulthood, with middle-aged adults performing worse than young (Issacowitz et al., 2007; Mill et al., 2009). In contrast, the ability to decide between more complex emotion labels for pictures of eyes is maintained through middle-age, only showing declines after age 60 (Hartshorne \& Germine, 2015). Understanding sarcasm is a relatively complex social cognition task, so might be preserved into middle-age. Our first hypothesis was therefore that older adults would show specific impairments in the comprehension of sarcasm compared to younger and middle-aged counterparts.

As outlined above, there are different ways to convey sarcasm which may influence the cognitive and social perceptual load of understanding sarcastic intent. For example, paradoxical sarcasm is signaled via a clear mismatch between the content of speech and reality, while simple sarcasm depends more on picking up nonverbal cues to emotions and intentions (McDonald \& Flanagan, 2004). It can therefore be predicted that age differences would be greater on simple sarcasm, which depends mainly on decoding nonverbal cues to emotions and intentions, as compared to paradoxical sarcasm where there are more contextual cues to aid understanding of sarcastic intent. We explored this through the use of videos (McDonald et al., 2002) which showed both paradoxical and simple sarcasm. Also, it has been argued that interpretation of direct sarcasm (where meaning is directly reversed) involves fewer cognitive resources than understanding indirect sarcasm (where meaning is more subtly ironic: Bucciarelli et al., 2003). Age differences may therefore be greater for interpreting indirectly sarcastic statements than more direct ones, because the former more heavily loads cognitive resources. We 
tested this using variants of the written sarcasm stories described by Channon et al. (2007). Our second hypothesis was therefore that age differences would be greater in interpreting simple (compared to paradoxical) and indirect (compared to direct) sarcasm.

The ability to understand sarcasm is dependent on both emotion perception skills such as picking up affective cues from faces, voices and gestures and theory of mind skills to decode others' intentions. Previous studies indicate that problems with theory of mind and emotion perception are related to the ability to interpret sarcasm (e.g. Channon et al., 2005). Older adults have impairments in emotion perception and theory of mind, so these social cognitive skills might be important in determining any problems in sarcasm comprehension. We will therefore investigate the relationships between these variables. Previous studies indicate that emotion perception partially mediated age differences in other aspects of social judgment such as social gaffes and deceit detection (Halberstadt, et al., 2011; Ruffman, et al., 2012) as well as false belief reasoning (Phillips et al., 2011). We will follow up these results by exploring whether emotion perception specifically predicts age differences in sarcasm decoding. Our third hypothesis was that age differences in sarcasm comprehension would be mediated by problems with emotion perception and theory of mind.

We also explored the potential role of cognitive and emotional changes in old age. Given that online processing of emotions and intentions is likely to depend on working memory updating (e.g. Phillips et al., 2008), we tested whether age-related differences in sarcasm comprehension might be mediated by variance in working memory updating. Finally, given the importance of positivity biases in influencing social judgments in old age (Charles \& Carstensen, 2008) we looked at whether any age differences in interpreting sarcasm might be related to levels of positive and negative affect.

\section{Methods}

\section{Participants}


116 participants completed the tasks: 40 young adults aged between 18 and 39 ( 27 females), 40 middle-aged adults aged between 40 and 64 ( 21 females) and 36 older adults aged between 65 and 86 (19 females). Participants were recruited from local contacts and groups, and through the University community, and were given a small payment for the testing session. Participants reported that they had no current neurological or psychiatric illness. Older adults also completed the MMSE test (Folstein, Folstein \& McHugh, 1975). All scored above 26 (range 27-30, $M=28.77, \mathrm{SD}=1.09$ ), which is the suggested cut-off for possible dementia in a well-educated sample (Mitchell, 2009). Descriptive statistics for age differences in years of education, Mill Hill vocabulary scores (Raven, Raven, \& Court, 1998) and health self-ratings on a scale of 1-9 are shown in Table 1.The three age groups differed in years of education, $F(2,113)=6.21, p<.01, \eta_{p}^{2}=.10$, with Bonferroni tests revealing that older adults had fewer years of education than young or middle-aged participants. There were also age differences in performance on the Mill Hill vocabulary test, $F(2,113)=17.25, p<.001, \eta_{p}{ }^{2}=.24$, with middle-aged and older adults both performing better than younger adults. The three age groups did not differ in selfrated health, $F(2,113)=1.13, \eta_{p}^{2}=.02$.

\section{Stimuli and Procedure}

The tasks below were presented in one of two counterbalanced orders, as part of a larger battery of cognitive and emotional tasks. The testing session lasted for approximately two hours.

\section{Understanding sarcasm from videos.}

Participants completed the Social Inference - Minimal subtest of The Awareness of Social Inference Test (TASIT, McDonald et al., 2002). This test consists of 15 videos of acted social interactions between 20-60s long, in which five sincere, five simple sarcasm and five paradoxical sarcasm exchanges are shown. Four questions are presented after each video, which ask for specific details about what one of the people in the video was doing, thinking and feeling, and what meaning they were trying to communicate to the other person. For example, in one simple sarcasm video a woman is busily doing a 
domestic task while a man reads a book and she says (sarcastically) 'Are you busy - I know you've got a lot on?' Participants are asked to answer yes or no to the following questions (correct answer in brackets): A. Is she is trying to pressure him into helping her? (yes); B.Is she trying to say it's OK if he doesn't help? (no); C. Does she think he should help her?(yes); D. Is she annoyed with him? (yes). In both sincere and simple sarcasm vignettes the meaning of the script is neutral so that paralinguistic cues must be used in order to interpret the meaning. In the paradoxical sarcasm videos the meaning only makes sense if interpreted as sarcastic, so there are semantic cues to non-literal meaning. Performance was scored by adding together the scores for the four questions for each video. For each condition (sincere, simple sarcasm, paradoxical sarcasm) the maximum total score was therefore 20 . These scores were converted into percentage correct. For each question, response time was also recorded, but these were not further analyzed.

\section{Understanding sarcasm from stories.}

The stories used were adapted from Channon et al. (2007). There were ten sarcastic stories, five of which involved direct sarcasm and five indirect sarcasm. There were also ten control stories which did not involve any sarcasm. Five of these involved the attribution of some kind of mental state to a protagonist, while five involved a physical event. Examples of each story type are given in Appendix 1. For each of the vignettes, the text was presented sentence by sentence on a computer screen. Participants could look at each sentence as long as they wished, and then pressed a button to progress to the next. Each story involved six sentences, and the stories in the different categories were matched for length. We presented the information in this way in order to more closely match the way in which social information is processed in everyday life (i.e. online and without the ability to keep referring back to previous information). Moreover, pilot work revealed that performance on the task was close to ceiling in both young and older adults if full stories were presented with questions below. 
After presentation of each final sentence participants were asked a question about what happened in the story, with four multiple choice answers to choose from. For example, in a direct sarcasm story about a woman taking her friend (Jean) to see a play which turned out to be not very good, Jean said "That was a fantastic play you took me to see!" The question was: What did Jean mean when she said that? Possible answers to choose from were: (a) That play was terrible (correct answer); (b) That was a very good play; (c) Next time I'll check what the critics say; (d) That was a beautiful theatre. Participants were told that more than one answer might appear correct, but that they should choose the best response which most fully answered the question. Reaction times to each screen presentation were recorded, but are not further analyzed here.

Sarcasm stories involved questions about the meaning of a protagonist's statement, while mental state control stories involved understanding other aspects of the mental state of a protagonist. Physical control stories asked about why a particular event had occurred and did not require any mental state attribution. Performance for each type of story was scored in terms of percentage accuracy. Participants were asked a further memory question about each story, but performance on this measure was close to ceiling, and did not differ across the age groups.

\section{Measure of Emotion Perception: Dynamic Faces task.}

In order to assess emotion perception skills, photographs from the Facial Emotion Expression Stimuli and Test (Young, Perrett, Calder, Sprengelmeyer \& Ekman, 2002) were used. Photographs depicting each of the six basic emotions from the stimulus set were used to create dynamic stimuli. Dynamic stimuli were used as these more accurately resemble the way in which emotions are experienced in everyday life, when expressions develop across time and are often fleeting (Ambadar, Schooler \& Cohn, 2005). For each face, dynamic stimuli were prepared which blended photographs of neutral, $25 \%, 50 \%, 75 \%$ and $100 \%$ emotional intensity into a 6 s animation. Each video began with a neutral face, and then developed to a maximum emotional intensity and then returned to neutral again. 
There were 72 videos (twelve for each of the six basic emotions: anger, disgust, fear, happiness, sadness, surprise), and during each video the participant was presented with a list of the possible response options from which they had to choose a label.

\section{Measure of Theory of Mind: the Faux Pas task.}

In order to assess theory of mind skills the Faux Pas task was used (Stone, Baron-Cohen \& Knight, 1998). This task required participants to identify whether or not a person described in a verbal vignette had committed a social faux pas. This requires the understanding and application of social rules in order to decide whether a statement is inappropriate and violates a social norm. Good task performance requires that participants simultaneously understand the mental states of two protagonists: the person making the faux pas who does not realize they have said the wrong thing, and also the listener whose feelings are being hurt (Stone et al., 1998). We used this measure to assess theory of mind because we wanted a task which was (a) verbal and (b) demanded understanding of intentions and emotions in a social context. Given that we have both verbal and visual sarcasm tasks, it was important to look at both verbal (theory of mind) and visual (emotion perception) potential mediators. When deciding which verbal theory of mind task to use we considered the most widely used adult test (variants of the Strange Stories test, e.g. Happé et al., 1998). However this task contains multiple items assessing understanding of non-literal meanings (including sarcasm, deception and white lies) so we felt it was too conceptually close to our sarcasm measures to be a more general measure of theory of mind in this case. Measures of faux pas understanding have been successfully used to assess theory of mind in adult aging studies (e.g. Halberstadt, et al. 2011; MacPherson, Phillips \& Della Sala, 2002).

Participants read a series of short stories, 10 of which contained a faux pas and 10 control stories that did not. Each story was presented in full on a computer screen and participants could spend as long reading the story as they wished. The story was followed by an initial faux pas detection question which asked whether someone in the story said something they should not have said. If participants responded 
'yes' to this question they were asked three further questions: Who said something inappropriate? Why was it inappropriate? Why did they say it? The accuracy of faux pas understanding was calculated by looking at responses to the initial faux pas question for each of the twenty stories, summing the 'yes' answers to faux pas stories and 'no' answers to control questions (maximum score $=20$ ). The accuracy of mental state understanding was calculated by adding together correct answers for each of the faux pas stories on the three further questions asking about who and why (maximum score $=30$ ). An overall score on the test was calculated by adding together the detection and mental state understanding scores and converting the resulting score (out of 50) into percentage accuracy.

\section{Measure of working memory: the $n$-back task.}

The N-back task was adapted from Braver et al. (1997) to investigate a participant's ability to update and monitor information in working memory. This task involved the presentation of a sequence of numbers (ranging from 1 to 9) individually in the center of the screen. During a 1-back practice block of trials, participants had to monitor stimuli and decide whether the presented number was the same as the number that had been presented immediately before by pressing one of two keys labeled 'yes' and 'no' on a response box. The 2-back updating condition consisted of 38 trials where participants had to report whether the number presented was the same as the number before the last (presented 2 trials before it) by pressing either a yes or no key. Each stimulus remained on screen until the participant had made a response. This was then followed by an inter-stimulus interval of $800 \mathrm{~ms}$. Percentage accuracy on the 2-back condition was used as the dependent measure in the current study. Note that 31 of the young, 38 of the middle-aged and 33 of the older adults completed the N-back task.

\section{Measure of current emotion.}

We used the 10-item short Positive and Negative Affect Schedule (PANAS, Watson, Clark \& Tellegen, 1988) to assess current positive and negative emotional state.

\section{Results}


In order to test the hypothesis that age differences would be greater on understanding sarcastic compared to sincere exchanges separate ANOVAs were carried out for video and story tasks. These analyses also tested the hypothesis that age effects would be greater for simple (compared to paradoxical) sarcasm for the video task, and indirect (compared to direct) sarcasm for the stories task.

\section{Videos of sincere and sarcastic exchanges}

The effects of age and video type were explored with a 3 (age group) x 3 video type (sincere, simple sarcasm, paradoxical sarcasm) mixed design analysis of variance (ANOVA). Age was a betweensubjects factor and video type was a within-subjects factor, and the dependent variable was accuracy of performance on the sarcasm task. There was a significant effect of group, $F(2,113)=10.71, p<.001, \eta_{p}{ }^{2}$ $=.16$. Bonferroni tests with $p<.05$ revealed that older adults $(M=79.72)$ were less accurate than both young $(M=86.42)$ and middle-aged participants $(M=89.54)$. There was an effect of video type, $F(2$, 226) $=13.56, p<.001, \eta_{\mathrm{p}}{ }^{2}=.11$, and paired-samples $\mathrm{t}$-tests revealed that performance on paradoxical sarcasm was higher $(M=90.04)$ than on sincere $(M=81.55)$ or simple sarcasm videos $(M=84.66)$. There was also an interaction between age group and type of video, $F(4,226)=2.79, p<.05, \eta_{p}{ }^{2}=.05$, see Figure 1. The interaction was explored through pairwise comparisons with Bonferroni adjustment. For the sincere videos, there was no effect of age. For the simple sarcasm videos, there were significant age effects, with younger $(p<.01)$ and middle-aged $(p<.001)$ participants outperforming older adults. Also for the paradoxical sarcasm videos younger $(p<.05)$ and middle-aged participants $(p<.001)$ outperformed older adults. These results support the hypothesis that older adults would have difficulty in interpreting sarcastic, but not sincere exchanges.

To check whether the age differences in interpreting sarcasm were greater for simple compared to paradoxical sarcasm a separate $3 \times 2$ ANOVA was carried out looking at the effects of age group and sarcastic video type (simple v paradoxical). There was no interaction between age group and sarcasm 
type, $F(1,112)=1.58, p=.21, \eta_{p}^{2}=.03$. This does not support the hypothesis of greater age differences on the simple sarcasm videos.

\section{Control and sarcasm stories}

The effects of age and story type were explored with a 3 (age group) $x 4$ story type (direct sarcasm, indirect sarcasm, mental control, physical control) mixed design ANOVA. Age was a betweensubjects factor and story type was a within-subjects factor, and the dependent variable was accuracy of performance on the sarcasm task. There was a significant effect of group, $F(2,113)=6.94, p=.001, \eta_{p}{ }^{2}$ $=.11$. Bonferroni tests revealed that older adults $(M=90.56)$ were less accurate than both young $(M=$ 94.50) and middle-aged participants $(M=95.50)$. There was an effect of story type, $F(3,339)=21.30, p$ $<.001, \eta_{p}{ }^{2}=.16$, with performance on the physical control items $(M=98.21)$ higher than on indirect sarcasm $(M=95.41)$, which in turn was performed better than both mental control $(M=91.55)$ and direct sarcasm stories $(M=88.91)$. There was also an interaction between age group and type of story, $F$ $(6,339)=3.75, p<.01, \eta_{p}^{2}=06$, see Figure 2 . To explore that interaction further, pairwise comparisons with Bonferroni adjustment were carried out. There was no effect of age group on either mental or physical control stories. For the direct sarcasm stories young $(p<.01)$ and middle-aged participants $(p<$ .05) outperformed old. For the indirect sarcasm stories the middle-aged group were significantly better than the older group $(p<.01)$, but neither group significantly differed from the young group. These results support the prediction that older adults would have difficulty in interpreting sarcastic but not control stories.

To check whether the age differences in interpreting sarcasm were greater for indirect compared to direct sarcasm a separate $3 \times 2$ ANOVA was carried out looking at the effects of age group and sarcasm story type (direct $v$ indirect). There was no interaction between age group and sarcasm type, $F$ $(1,112)=2.29, p=.11, \eta_{p}^{2}=.04$. This does not support the hypothesis that age effects would be greater for the indirect sarcasm stories. 


\section{The possible role of emotion perception, theory of mind and working memory.}

The next set of analyses tested the hypothesis that other key measures might mediate the effects of age on sarcasm comprehension. First, age effects in these proposed mediators (emotion perception, theory of mind and working memory) were assessed, and second, correlations between the proposed mediators and sarcasm comprehension were explored. Where both age effects and correlations between the mediator and sarcasm were significant, mediation models were tested.

Table 2 describes age group differences in emotion perception (EP), theory of mind (ToM), working memory (2-back) and current affect (PANAS). One-way ANOVAs revealed a significant age group effect on $E P, F(2,112)=8.34, p<.001, \eta_{p}{ }^{2}=.12$, with Bonferroni tests showing that the older group performed worse than young $(p<.01)$ and middle-aged $(p<.001)$. There was no age group effect on ToM performance, $F(2,112)=2.34, p=.10, \eta_{p}{ }^{2}=.04$. A significant age group difference in 2-back working memory $(\mathrm{WM})$ performance, $F(2,101)=5.12, p<.01, \eta_{\mathrm{p}}{ }^{2}=.13$, was due to the older group performing worse than the young $(p<.001)$. Older adults had lower levels of negative affect than both younger $(p<.001)$ and middle-aged counterparts $(p<.01), F(2,101)=9.23, p<.001, \eta_{p}{ }^{2}=.16$. Age differences in positive affect, $\mathrm{F}(2,101)=4.79, p<.05, \eta_{\mathrm{p}}{ }^{2}=.09$, were due to older adult having higher levels of positive affect than middle-aged adults $(p<.01)$.

Table 2 also shows the correlations between performance on the sarcasm comprehension tasks and measures of ToM, EP and WM and current emotion. Note that performance on the different types of sarcastic video was combined (simple and paradoxical) as there was no indication of differential age effects, and the same applied to the direct and indirect sarcasm stories. Both ToM and EP were correlated with the ability to understand sarcasm from both stories and videos. However, as performance on the ToM test was not affected by age it could not be considered as a possible mediator for the age-sarcasm relationship. We therefore tested mediation models to explore whether EP might statistically mediate shared variance between age and sarcasm understanding from (1) videos and (2) 
stories. WM performance measured by the 2-back task did not correlate with sarcasm comprehension from the videos, while the correlation approached significance for the story task. We therefore tested a third mediation model to explore whether WM might mediate shared variance between age and the stories sarcasm task. There were no significant correlations between measures of current positive or negative affect and sarcasm comprehension.

Mediation analyses were carried out following the methods outlined by Preacher and Hayes $(2004,2008)$. In mediation analysis a series of regressions are carried out to test whether the association between the predictor variable (age group) and the dependent variable (sarcasm comprehension) is significantly mediated by a third variable (in this case EP or WM). An initial regression examines the relationship between the predictor and mediator variable (path $a$ in Figure 3 ). The second step confirms the relationship between the predictor variable and the dependent variable (path $c$ in Figure 3 ). The final regression analysis simultaneously enters the mediator and predictor variables to explain variance in the dependent variable (paths $b$ and $c^{\prime}$ in Figure 3). Reductions from $c$ to $c^{\prime}$, once the mediator is entered, suggest a possible mediation effect. The significance of any mediation effect can be tested using bootstrap procedures (Preacher \& Hayes, 2004, 2008: see http://www.afhayes.com/ for the macros used). Note that we used age group and not age in years as a predictor because of non-linear relationships between age and sarcasm decoding.

In the first mediation analysis the influence of EP on age group differences in understanding sarcasm from videos was investigated. EP was a significant predictor of sarcasm comprehension when entered along with age in the analysis (see Figure 3i). Bootstrapping revealed that emotion perception was a significant mediator of the age-sarcasm relationship (bias corrected confidence intervals with 5000 resamples from -2.600 to -0.029 , do not include zero, $p<.05$ ). Note that this is only a partial mediation effect as the age-sarcasm relationship remained significant (see $c^{\prime}$ in Figure 3i). 
In the second mediation analysis (see Figure 3ii) the influence of EP on age group differences in understanding sarcasm from stories was tested. However, EP was not a significant predictor of sarcasm comprehension when entered in regression with age (see b in Figure 3ii), and bootstrapping confirmed that no mediation effect was present (confidence intervals from -0.621 to 0.367 include zero). These results partially support the prediction that emotion perception would mediate age differences in sarcasm comprehension: the mediation effects were found only in the videos task.

A final analysis looked at whether age differences in decoding sarcasm from stories might be mediated by WM scores from the 2-back task (see Figure 4). WM was not a significant predictor of sarcasm comprehension when entered in regression with age, and bootstrapping confirmed that no mediation effect was present (confidence intervals from -1.199 to 0.330 include zero). This result does not support the idea that working memory problems mediate age differences in sarcasm understanding.

\section{Discussion}

\section{The pattern of age differences in understanding sarcasm}

In line with other social cognition differences across the lifespan, this study indicated that older adults were less likely to understand sarcasm from both story and video stimuli. Most aging studies of social cognitive skills employ an extreme groups design, but the current study recruited a lifespan adult sample including a middle-aged group. Supporting previous findings on mental belief reasoning skills (Phillips et al., 2011) the middle-aged group in the current study performed similarly to the younger group. While there are relatively early age-related declines in some social perception skills such as basic emotion perception (Mill et al., 2009), the current results indicate that more complex social cognitive skills such as comprehending sarcastic intent are spared until later in the lifespan. This may reflect relatively early age-related change in perceptual mechanisms (which are important in some tasks of facial emotion perception) whereas age effects on more complex and context dependent tasks such as sarcasm detection or belief reasoning might occur later in the lifespan. However, it should be noted that 
there was no evidence of the middle-aged group performing worse than the young on the emotion perception task in the current study. The trajectory of adult age effects on different aspects of social cognition may be highly task-specific, depending on perceptual and cognitive demands, the role of wisdom and experience, and social and emotional demands of different task conditions.

There was clear evidence for specificity of age-related difficulty in understanding sarcasm: no agerelated differences were found in the control conditions for the videos or stories. This concurs with previous research on age-related changes in social cognitive tasks, where age differences are greater on emotion judgments compared to tasks requiring age or object judgments (Ruffman et al., 2012; Sullivan \& Ruffman, 2004). A recent meta-analysis of a broad range of theory of mind tasks indicates that age differences are greater in mental state decoding than matched control tasks (Henry et al., 2013).

One aim of this study was to look at the effects of aging on understanding different types of sarcasm. In the videos task there were equivalent age effects in interpreting simple sarcasm (which was dependent on picking up nonverbal cues to intention), and paradoxical sarcasm (which was more reliant on understanding semantic meaning). While paradoxical sarcasm in these videos is conveyed mainly by a mismatch between the situational context and the statement made, inspection of the videos reveals that there are also nonverbal cues from facial expression and tone of voice to indicate non-literal intent. Performance on the video task indicated that paradoxical sarcasm was understood better than simple sarcasm, suggesting that it was easier to interpret: this may reflect the fact there are both contextual and nonverbal cues to signal sarcastic intent. One unexpected result was that understanding of the sincere videos was at a lower level than the sarcastic videos. A similar result has been reported in other healthy control samples (e.g. McDonald et al., 2002). Lower performance on sincere as compared to sarcastic videos in the current sample might reflect a bias for participants to suspect non-literal meanings, given that the test is comprised of a majority of scenarios where sarcasm is present. It would be useful in future to develop stimuli which have a lower frequency of sarcastic statements. 
Performance on the stories sarcasm task did not support the prediction that age differences would be more apparent on indirect than direct sarcasm vignettes: there was not a significant interaction between age and type of sarcasm. However paired comparisons revealed a slightly different age pattern on direct and indirect sarcasm stories. Older adults performed worse than young and middle-aged on the direct sarcasm trials, where a protagonist makes a remark with directly opposite meaning to the intention. For the indirect sarcasm stories, where the sarcastic remark was more subtle and not the direct opposite of the intended meaning, middle-aged adults performed better than old, but the young group did not differ from either. Younger adults may not perform particularly well on these specific items because they are of higher social and semantic complexity than the more direct forms of sarcasm. Understanding of direct sarcasm develops earlier in childhood than indirect (Dews et al., 1996), and it may be that interpreting complex meanings peaks in middle-age. However, this result may be confounded by ceiling effects: the middle-aged group performed at $98 \%$ accuracy on these trials. There were not significant age differences on either type of control story, and in fact there was a trend for older adults to perform better than young on the physical control stories. This indicates that older adults were specifically having difficulty in making non-literal interpretations of speech rather than struggling to process the verbal material online more generally.

\section{The role of emotion perception and theory of mind}

We also investigated the potential role of emotion perception and theory of mind in age differences in understanding sarcasm. Emotion perception was related to both age and comprehension of sarcasm, so mediation analyses were carried out. These indicated that for the video task age differences in comprehension of sarcasm were partly mediated by emotion perception. Problems with perceiving nonverbal cues such as emotions are important in detecting and understanding non-literal intent from speech, and this is more important when visual information is available. The current results fit with previous studies indicating the potential importance of emotion perception in decoding sarcastic 
intent in neurological populations (e.g. Kipps et al., 2009; Rankin et al., 2009; Shamay-Tsoory et al., 2005), as well as findings that detecting lies and social gaffes in old age may be partly mediated by poorer emotion perception (Halberstadt et al., 2011; Ruffman et al., 2012.). The finding that emotion perception partially mediated age-related variance in another social cognition skill suggests overlap in the developmental processes involved in social cognition. Conceptually, this indicates that one of the important influences on age-related changes in relatively complex social cognition tasks such as sarcasm comprehension may be more basic social perception processes, which are also subject to age-related change. However, it should be noted that the emotion perception task used here was not a naturalistic task, as it involved manipulation of static faces to produce dynamic clips. It would be useful in future research to use real videos which portray the true time course of emotions unfolding, and also to use tasks which extend beyond facial expressions to also include tone of voice and bodily gestures.

Age differences in interpreting sarcasm from stories were not mediated by our current visual measure of emotion perception. This contrasts with a previous finding (Phillips et al., 2011) that age differences in interpreting false beliefs from both stories and videos were mediated by the ability to extract emotional information from whole body stimuli. A different set of both mediator (faces $v$ whole bodies) and dependent variables (sarcasm comprehension $v$ false belief reasoning) were used in these studies, and it is important for future research to explore in more detail whether age differences in a range of social inference tasks relates to multiple aspects of emotion perception. This will shed some more light on the important conceptual issue of how different aspects of social cognition are interdependent, and the extent to which more basic social perception processes are essential for more complex social inference.

While the faux pas test of theory of mind was correlated with sarcasm, there were no effects of age on understanding faux pas. Previous studies have reported mixed effects of age on understanding of social faux pas, with some reporting no age effect in the accuracy of detection (MacPherson et al., 
2002), while others indicate age-related decline (Halberstadt et al., 2011). Stanley, Lohani and Issacowitz (2014) report that older adults find faux pas less funny than young and middle-aged adults , while Zhang, Fung, Stanley, Issacowitz \& Ho (2013) reported that older adults had poorer understanding of faux pas only when the experimenter was unknown to the participant. Because there were no age relationships with the faux pas task used here we could not further explore whether theory of mind might mediate age differences in sarcasm comprehension.

\section{Cognitive components of understanding sarcasm}

We also tested whether age-related differences in sarcasm comprehension might be mediated by declining working memory. There were significant age differences in working memory in our sample, and performance on the working memory task was weakly correlated with understanding sarcasm from the stories (with $p=.057$ ). The relationship between working memory and sarcasm comprehension from videos was not significant. A mediation analysis indicated that the age differences in understanding sarcasm from stories could not be explained in terms of declining working memory. Working memory is therefore unlikely to be the major factor underlying age differences in decoding sarcasm. This contrasts with previous findings that age differences in belief reasoning were partially explained by working memory updating (Phillips et al., 2011). Despite the sequential nature of both the story and video sarcasm measures used in the current study, the requirement to hold in mind contextual information and protagonists' intentions did not seem to overload participants' working memory systems. Conceptually this indicates that age differences in social cognitive skills such as sarcasm comprehension are distinct from more traditional cognitive resources. It should be noted that we only have one (verbal) measure of working memory here, and it would be useful in future studies to use a range of memory updating tasks.

While we assessed working memory updating, we did not assess other aspects of executive function which might be important in social cognition tasks such as inhibition and switching. In the 
current tasks, to decide whether a protagonist was being sarcastic required the consideration of both literal and non-literal interpretations of a statement. In common with many social cognition tasks (such as false belief reasoning or detecting deception), this requires the resolution of discrepant information, which might load control processes such as inhibition and switching. Previous studies have produced mixed results on whether age differences in the resolution of discrepant belief states are mediated by inhibition (Bailey \& Henry, 2008; Phillips et al., 2011). This likely reflects difficulties in assessing the construct of inhibition (Bailey \& Henry, 2008), which is not a unitary construct.

There are many situations in which social cues are mismatched and so potentially complex to resolve. For example older adults have increased difficulties when conflicting emotional cues are presented (Hunter, Phillips \& MacPherson, 2010). It would be of interest to explore whether different channels of information are more dominant as people get older. For example, in the current study, older people might rely more on the meaning of what people say rather than placing weight on the contextual and pragmatic cues which indicate sarcastic intention. This might occur because of a bias towards literal interpretations or increased self-doubt about interpreting emotional cues. Also, awareness of agerelated change in hearing could potentially lead to an increased reliance on visual cues.

\section{Emotional, social and cultural influences on interpreting sarcasm}

It is important to note that another conceptual interpretation of the age differences in attributing sarcastic intention is that they reflect positivity biases in old age (Reed et al., 2014). Older adults are more likely to ignore negative social information and reappraise negative situations in a more positive light (e.g. Charles \& Carstensen, 2008). This might mean that older people are less alert to negative intent, such as sarcasm. In our sample older adults reported lower levels of negative affect than young and middle-aged participants, and higher levels of positive affect compared to middle-aged. However there were no relationships between measures of current affect and performance on any of the sarcasm measures. In order to explore this issue in more detail it would be interesting to directly compare age 
effects in understanding sarcasm with understanding of more positive intents which also involve nonliteral interpretations. An example might be picking up on 'white lies' where the speaker has deceptive intent, but with positive motives to spare someone's feelings.

Our sample was not matched across the age groups for either vocabulary levels or years of education. Additional analyses covarying these factors did not change the results reported above concerning age differences. Another factor which could contribute to current findings is generational differences in use of (or familiarity with) sarcasm. There is a stereotype that recent generations use irony and sarcasm more frequently than previous generations do. However there is not much empirical evidence to determine whether this reflects reality. In contrast to the idea that older adults rarely use sarcasm, Liptak, Tate, Flatt, Oakley \& Lingler (2014) report that in social interactions between older adults with cognitive impairment and their caregivers the most pervasive type of humor was sarcasm or dry wit. The use of sarcasm was often affectionate and was argued to provide a release of tension. Exploring age differences in the use of sarcasm in interpersonal interaction with close friends or family could indicate whether shared affectionate humor might have a positive function.

Cultural differences may also be important. In the UK sarcasm has been a staple of humor for many decades, and it has been argued that behavior which would be seen as aggressive or denigrating in the US would be seen as funny in the UK (Vernon, Martin, Aitken Schermer, Cherkas \& Spector, 2008). Indeed Vernon et al. point out that the North American version of 'The Office' depicted a character more sensitive and tolerant than his UK counterpart. In Scotland, where the current research was carried out, much humor is traditionally based on irony and mockery (Francesconi, 2011), and this often takes the form of affectionate but sarcastic comments. In North American samples older adults are less likely to endorse aggressive humor than young, and this mediates age differences in perceived appropriateness of social behavior (Stanley et al., 2014): but it is not clear if this would apply to a UK sample. While there is no direct evidence on cultural differences in reaction to sarcasm in older adults, 
there is relevant evidence for children. Canadian and Czech children react very differently to sarcastic praise, probably because there is differential use of sarcastic praise in everyday speech (Filippova, 2014). Also, Iranian children show greater understanding of sarcasm than age-matched Australian children (Shahaeian, Nielsen, Peterson \& Slaughter, 2014). The current results could therefore be influenced by cultural effects: this should be explored in future through data collection in multiple cultures.

\section{Directions for future research}

Future research should explore whether there are age differences in the frequency of encountering and using sarcasm in everyday life, and how this relates to more generally cynical attitudes, positivity biases, and indicators of cognitive and social skills. Channon et al. (2005) describe a rating scale to assess participants' likelihood of being sarcastic in different situations - whereas it might be appropriate to use affectionate sarcasm with a family member, it would be less appropriate in a more formal situation such as a job interview. It would also be interesting to carry out linguistic analyses of social interactions or existing prose written by authors of different ages. Generational differences could be explored by looking at examples of sarcastic humor (cartoons, radio or TV programs) from different decades. In the current study the age of the people in the stories/videos was not controlled, and given evidence of own-age biases in response to some social stimuli (e.g. Slessor, Phillips, Ruffman, Bailey \& Insch, 2014) it would be useful to explore this issue in future studies. However, an own-age advantage in social perception is not seen in all tasks: for example, in identifying emotions from older faces (e.g. Ebner, He \& Johnson, 2011): instead older faces are more difficult to interpret for young and older participants (Fölster, Hess \& Werheid, 2014).

In the last decade there has been increasing evidence that adult aging can impair both relatively simple tasks of social perception (e.g. following eye gaze, see Phillips et al., 2014 for a review) and more complex measures of social reasoning (e.g. false belief understanding, see Henry et al., 2013 for a review). However, some aspects of social understanding may improve across the lifespan, such as 
interpreting videos of smiles (Murphy, Lehrfeld \& Isaacowitz, 2010), and empathy (Richter \& Kunzmann, 2011). In order to develop a more detailed theoretical model of what might underlie age differences in a range of social skills, it would be valuable to have larger scale studies which include a range of social perception and social understanding tasks, as well as more detailed measures of cognition. However there is not an overarching model of these social cognitive processes to aid appropriate selection of tasks. While there have been important distinctions made between hot and cold aspects of social cognition (e.g. Shamay-Tsoory \& Aharon-Peretz, 2007), modality of presentation of stimuli such as verbal versus visual (Slessor et al., 2007), and implicit versus explicit measures (Bailey \& Henry, 2009) it is not clear which of these dimensions are most important to consider when assessing age differences.

Given the evidence of non-linear age effects, it would be particularly useful to learn more about the performance of middle-aged people in relation to younger and older adults, as well as more about how the oldest old perform on these tasks. It is important to understand more about the influence of aging on social cognition tasks such as sarcasm comprehension, because failures to understand others' intentions could cause misunderstandings in social interaction. There is evidence that problems with decoding sarcasm can predict poorer social interaction skills in a group of patients with brain injury (McDonald, Flanagan, Martin, \& Saunders, 2004). Future studies should address whether age differences in interpreting non-literal statements such as sarcasm influence intergenerational communication. Age differences in the nuanced interpretation of meaning could impede shared understanding.

\section{Conclusions}

In conclusion, the current research indicates that older adults have problems in decoding different types of sarcasm from both written and video formats. These age effects were specific, as indicated by the lack of age effect on matched control tasks. Mediation analyses indicated that difficulties with emotion perception may contribute to these age effects. Older adults may be prone to more literal interpretation of sarcastic statements, with possible consequences for social interactions. 


\section{References}

Ambadar, Z., Schooler, J. W., \& Cohn, J. F. (2005). Deciphering the enigmatic face: The importance of facial dynamics in interpreting subtle facial expressions. Psychological Science, 16, 403-410. doi: 10.1111/j.0956-7976.2005.01548.x

Bailey, P. E., \& Henry, J. D. (2008). Growing less empathic with age: Disinhibition of the self-perspective. Journal of Gerontology: Psychological Sciences and Social Sciences, 63, 219-226. doi: 10.1093/geronb/63.4.P219

Bailey, P. E., \& Henry, J. D. (2009). Subconscious facial expression mimicry is preserved in older adulthood. Psychology and Aging, 24, 995-1000. doi: 10.1037/a0015789.

Bond, G. D., Thompson, L. A.,. \& Malloy, D. M. (2005). Vulnerability of older adults to deception in prison and nonprison contexts. Psychology and Aging, 20, 60-70. ddoi: 10.1037/0882-7974.20.1.60.

Braver, T. S., Cohen, J. D., Nystrom, L. E., Jonides, J., Smith, E. E., and Noll, D. C. (1997). A parametric study of prefrontal cortex involvement in human working memory. Neuroimage, 5, 49-62. doi : 10.1006/nimg.1996.0247

Bucciarelli, M., Colle, L., \& Bara, B. G. (2003). How children comprehend speech acts and communicative gestures. Journal of Pragmatics, 35, 207-241. doi: 10.1016/S0378-2166(02)00099-1

Bull, R., Phillips, L. H. \& Conway, C. A. (2008). The role of control functions in mentalizing: Dual task studies of Theory of Mind and executive function. Cognition, 107, 663-672. doi: 10.1016/j.cognition.2007.07.015

Champagne, M., Jean-Louis, S., \& Joanette, Y. (2006). Effect of aging on the processing of non-literal language. Canadian Journal on Aging- Revue Canadienne Du Vieillissement, 25, 55-64. doi: 10.1353/cja.2006.0020

Channon, S., Pellijeff, A., \& Rule, A. (2005). Social cognition after head injury: Sarcasm and theory of mind. Brain and Language, 93, 123-134. doi: 10.1016/j.bandl.2004.09.002 
Channon, S., Rule, A., Maudgil, D., Martinos, M., Pellijeff, A., Frankl, J., ... Shieff, C. (2007). Interpretation of mentalistic actions and sarcastic remarks: Effects of frontal and posterior lesions on mentalizing. Neuropsychologia, 45, 1725-1734. doi: 10.1016/j.neuropsychologia.2006.12.021

Charles, S. T.,. \& Carstensen, L. L. (2008). Unpleasant situations elicit different emotional responses in younger and older adults. Psychology and Aging, 23, 495-504. doi: 10.1037//a0013284

Charlton, R. A., Barrick, T. R., Narkus, H. S., \& Morris, R. G. (2009). Theory of mind associations with other cognitive functions and brain imaging in normal aging. Psychology and Aging, 24, 338348. doi: $10.1037 / \mathrm{a} 0015225$

Dews, S., Winner, E., Kaplan, J., Rosenblatt, E., Hunt, M., Lim, K., ... Smarsh, B. (1996). Children's understanding of the meaning and functions of verbal irony. Child Development, 67, 30713085. doi: $10.2307 / 1131767$

Ebner, N. C., He, Y. I., \& Johnson, M. K. (2011). Age and emotion affect how we look at a face: Visual scan patterns differ for own-age versus other-age emotional faces. Cognition and Emotion, 25, 983997.

Filippova, E. (2014). Developing appreciation of irony in Canadian and Czech discourse. Journal of Pragmatics, 74, 209-233. doi: 10.1016/j.pragma.2014.09.003

Folstein, M. F., Folstein, S. E.,. \& McHugh, P. R. (1975). Mini-mental state. A practical method for grading the cognitive state of patients for the clinician. Journal of Psychiatry Research, 12, 189198.

Fölster, M., Hess, U.,. \& Werheid, K. (2014). Facial age affects emotional expression decoding. Frontiers in Psychology, 5, 1-13. doi: 10.3389/fpsyg.2014.00030

Francesconi, S. (2011). Multimodally expressed humour shaping Scottishness in tourist postcards. Journal of Tourism and Cultural Change, 9, 1-17. doi: 10.1080/14766825.2010.521561 
Halberstadt, J., Ruffman, T., Murray, J., Taumoepeau, M., \& Ryan, M. (2011). Emotion perception explains age-related differences in the perception of social gaffes. Psychology and Aging, 26, 133-136. doi: 10.1037/a0021366

Happé, F. G. E., Winner, E., \& Brownell, H. (1998). The getting of wisdom: Theory of mind in old age. Developmental Psychology, 34, 358-362. doi: 10.1037/0012-1649.34.2.358

Hartshorne, J. K.,. \& Germine, L. T. (2015). When does cognitive functioning peak? The asynchronous rise and fall of different cognitive abilities across the life span. Psychological Science, 26, 433443. doi: $10.1177 / 0956797614567339$

Henry, J. D., Phillips, L. H., Ruffman, T., \& Bailey, P. E. (2013). A meta-analytic review of age differences in theory of mind. Psychology and Aging, 28, 826-839. doi: 10.1037/a0030677

Hunter, E. M., Phillips, L. H., \& MacPherson, S. E. (2010). Effects of age on cross-modal emotion perception. Psychology and Aging, 25, 779-787. doi: 10.1037/a0020528

Isaacowitz, D. M., Loeckenhoff, C., Lane, R., Wright, R., Sechrest, L., Riedel, R., \& Costa, P. T. (2007). Age differences in recognition of emotion in lexical stimuli and facial expressions. Psychology and Aging, 22, 147-259. doi: 10.1037/0882-7974.22.1.147

Kipps, C. M., Nestor, P. J., Acosta-Cabronero, J., Arnold, R., \& Hodges, J. R. (2009). Understanding social dysfunction in the behavioral variant of frontotemporal dementia: the role of emotion and sarcasm processing. Brain, 132, 592-603. doi: 10.1093/brain/awn314

Liptak, A., Tate, J., Flatt, J., Oakley, M. A.,. \& Lingler, J. (2014). Humor and laughter in persons with cognitive impairment and their caregivers. Journal of Holistic Nursing, 32, 25-34. doi: $10.1177 / 0898010113500075$

Luong, G.,. \& Charles, S. T. (2014). Age differences in affective and cardiovascular responses to a negative social interaction: The role of goals, appraisals, and emotion regulation. Developmental Psychology, 50, 1919-1930. doi: 10.1037/a0036621 
MacPherson, S., Phillips, L. H., \& Della Sala, S. (2002). Age, executive function and social decisionmaking: a dorsolateral prefrontal theory of cognitive aging. Psychology and Aging, 17, 598-609. doi: 10.1037/0882-7974.17.4.598

McDonald, S., \& Flanagan, S. (2004) Social perception deficits after Traumatic Brain Injury: The interaction between emotion recognition, mentalising ability and social communication. Neuropsychology, 18, 572-579. doi: 10.1037/0894-4105.18.3.572

McDonald, S., Flanagan, S., Martin, I., \& Saunders, C. (2004). The ecological validity of TASIT: A test of social perception. Neuropsychological Rehabilitation, 14, 285-302. doi: $10.1080 / 09602010343000237$

McDonald, S., Flanagan, S., \& Rollins, J. (2002). The Awareness of Social Inference Test (TASIT). Bury St Edmunds, UK: Thames Valley Test Company.

Mill, A., Allik, J., Realo, A., \& Valk, R. (2009). Age-related differences in emotion recognition ability: A cross-sectional study. Emotion, 9, 619-630.doi: 10.1037/a0016562

Mitchell, A. J. (2009). A meta-analysis of the accuracy of the Mini-Mental State Examination in the detection of dementia and mild cognitive impairment. Journal of Psychiatric Research, 43. 411431. doi: 10.1016/j.jpsychires.2008.04.014

Murphy, N. A., Lehrfeld, J. M., \& Isaacowitz, D. M. (2010). Recognition of posed and spontaneous dynamic smiles in young and older adults. Psychology and Aging, 25, 811-821. doi: $10.1037 / a 0019888$

Phillips, L. H., Bull, R., Allen, R., Insch, P., Burr, K., \& Ogg, W. (2011). Lifespan aging and belief reasoning: Influences of executive functions and social cue detection. Cognition, 120, 236-247. doi: 10.1016/j.cognition.2011.05.003

Phillips, L. H., Channon, S., Tunstall, M., Hedenstrom, A., \& Lyons, K. (2008). The role of working memory in decoding emotions. Emotion, 8, 184-191. doi: 10.1037/1528-3542.8.2.184 
Phillips, L. H., Slessor, G., Bailey, P. E., \& Henry, J. D. (2014). Older adults' perception of social and emotional cues. In Verhaeghen, P. \& Hertzog, C. (Eds). The Oxford handbook of emotion, social cognition, and everyday problem solving during adulthood (pp. 9-25). New York: Oxford University Press.

Preacher, K. J., \& Hayes, A. F. (2004). SPSS and SAS procedures for estimating indirect effects in simple mediation models. Behavior Research Methods, Instruments and Computers, 36, 717-731.

Preacher, K. J., \& Hayes, A. F. (2008). Asymptotic and resampling strategies for assessing and comparing indirect effects in multiple mediator models. Behavior Research Methods, 40, 879-891. doi: 10.3758/BRM.40.3.879

Rankin, K. P., Salazar, A., Gorno-Tempini, M. L., Sollberger, M., Wilson, S. M., Pavlic, D., ... Miller, B. L. (2009). Detecting sarcasm from paralinguistic cues: Anatomic and cognitive correlates in neurodegenerative disease. Neurolmage, 47, 2005-2015. doi: 10.1016/j.neuroimage.2009.05.077

Raven, J., Raven, J. C., \& Court, J. H. (1998). Manual for Raven's Progressive Matrices and Vocabulary Scales. San Antonio, TX: Harcourt Assessment.

Reed, A.E., Chan, L.,. \& Mikels, J.A. (2014). Meta-analysis of the age-related positivity effect: Age differences in preferences for positive over negative information. Psychology and Aging, 29, 115. doi: $10.1037 / \mathrm{a} 0035194$

Richter, D., \& Kunzmann, U. (2011). Age differences in three facets of empathy: Performance-based evidence. Psychology and Aging, 26, 60-70. doi: 10.1037/a0021138

Ruffman, T., Henry, J. D., Livingstone, V., \& Phillips, L. H. (2008). A meta-analytic review of emotion recognition and aging: Implications for neuropsychological models of aging. Neuroscience and Biobehavioral Reviews, 32, 863-881. doi: 10.1016/j.neubiorev.2008.01.001 
Ruffman, T., Murray, J., Halberstadt, J., \& Vater, T. (2012). Age-related differences in deception.

Psychology and Aging, 27, 543-549. doi: 10.1037/a0023380

Shahaeian, A. Nielsen, M., Peterson, C. C.,. \& Slaughter, V. (2014). Cultural and family influences on children's theory of mind development: A comparison of Australian and Iranian school-age children. Journal of Cross-Cultural Psychology, 45, 555-568. doi: 10.1177/0022022113513921

Shamay-Tsoory, S. G., \& Aharon-Peretz J. (2007). Dissociable prefrontal networks for cognitive and affective theory of mind: A lesion study. Neuropsychologia, 45, 3054-3067. doi: 10.1016/j.neuropsychologia.2007.05.021

Shamay-Tsoory, S. G., Tomer, R., \& Aharon-Peretz, J. (2005). The neuroanatomical basis of understanding sarcasm and its relationship to social cognition. Neuropsychology, 19, 288-300. doi: 10.1037/0894-4105.19.3.288

Shany-Ur, T., Poorzand, P., Grossman, S. N., Growdon, M. E., Jang, J. Y., Ketelle, R. S., Miller, B. L., \& Rankin, K.P. (2011). Comprehension of insincere communication in neurodegenerative disease: lies, sarcasm, and theory of mind. Cortex, 48, 1329-1341. doi: 10.1016/j.cortex.2011.08.003

Slessor, G., Phillips, L. H., \& Bull, R. (2007). Exploring the specificity of age differences in Theory of Mind tasks. Psychology and Aging, 22, 639-643. doi: 10.1037/0882-7974.22.3.639

Slessor, G., Phillips, L. H., \& Bull, R. (2008). Age-related declines in basic social perception: Evidence from tasks assessing eye-gaze processing. Psychology and Aging, 23, 812-822. doi: $10.1037 / \mathrm{a} 0014348$

Slessor, G., Phillips, L. H., Ruffman, T., Bailey, P. E., \& Insch, P. (2014). Exploring own-age biases in deception detection. Cognition and Emotion, 28, 493-506. doi:

$10.1080 / 02699931.2013 .839438$

Stanley, J. T., \& Blanchard-Fields, F. (2008). Challenges older adults face in detecting deceit: The role of emotion recognition. Psychology and Aging, 23, 24-32. doi: 10.1037/0882-7974.23.1.24 
Stanley, J. T., Lohani, M.,. \& Issacowitz, D. M. (2014). Age-related differences in judgments of inappropriate behavior are related to humor style preferences. Psychology and Aging, 29, 528541. doi: $10.1037 / a 0036666$

Sullivan, S., \& Ruffman, T. (2004). Emotion recognition deficits in the elderly. International Journal of Neuroscience, 114, 403-432. doi: 10.1080/00207450490270901

Stone, V. E., Baron-Cohen, S., \& Knight, R. T. (1998). Frontal lobe contribution to theory of mind. Journal of Cognitive Neuroscience, 10, 640-656. doi: 10.1162/089892998562942

Uekermann, J., Channon, S., \& Daum, I. (2006). Humor processing, mentalizing, and executive function in normal aging. Journal of the International Neuropsychological Society, 12, 184-191. doi: $10.1017 / \mathrm{S} 1355617706060280$

Uekermann, J., Thoma, P., \& Daum, I. (2008). Proverb interpretation changes in aging. Brain and Cognition, 67, 51-57. doi: 10.1016/j.bandc.2007.11.003

Vernon, P. A., Martin, R. A., Aitken Schermer, J., Cherkas, J. A. \& Spector, T. (2008). Genetic and environmental contributions to humor styles: A replication study. Twin Research and Human Genetics, 11, 44-47. doi: 10.1375/twin.11.1.44

Watson, D., Clark, L. A., \& Tellegen, A. (1988). Development and validation of brief measures of Positive and Negative affect: The PANAS Scales. Journal of Personality and Social Psychology, 47, 10631070. doi: $10.1037 / 0022-3514.54 .6 .1063$

Winner, E., Brownell, H., Happé, F., Blum, A., \& Pincus, D. (1998). Distinguishing lies from jokes: Theory of mind deficits and discourse interpretation in right hemisphere brain-damaged patients. Brain and Language, 62, 89-106. doi: 10.1006/brln.1997.1889

Young, A., Perrett, D., Calder, A., Sprengelmeyer, R., \& Ekman, P. (2002). Facial Expressions of Emotion Stimuli and Tests (FEEST) (Software Manual v2.1 ed.). Bury St Edmunds, England: Thames Valley Test Company. 
Zhang, X., Fung, H. H., Stanley, J. T., Isaacowitz, D. M., \& Ho, M. Y. (2013) Perspective taking in older age revisited: A motivational perspective. Developmental Psychology, 49, 1848-1858. doi: $10.1037 / \mathrm{a} 0031211$ 
Table 1:

Descriptive statistics for age group differences in background variables.

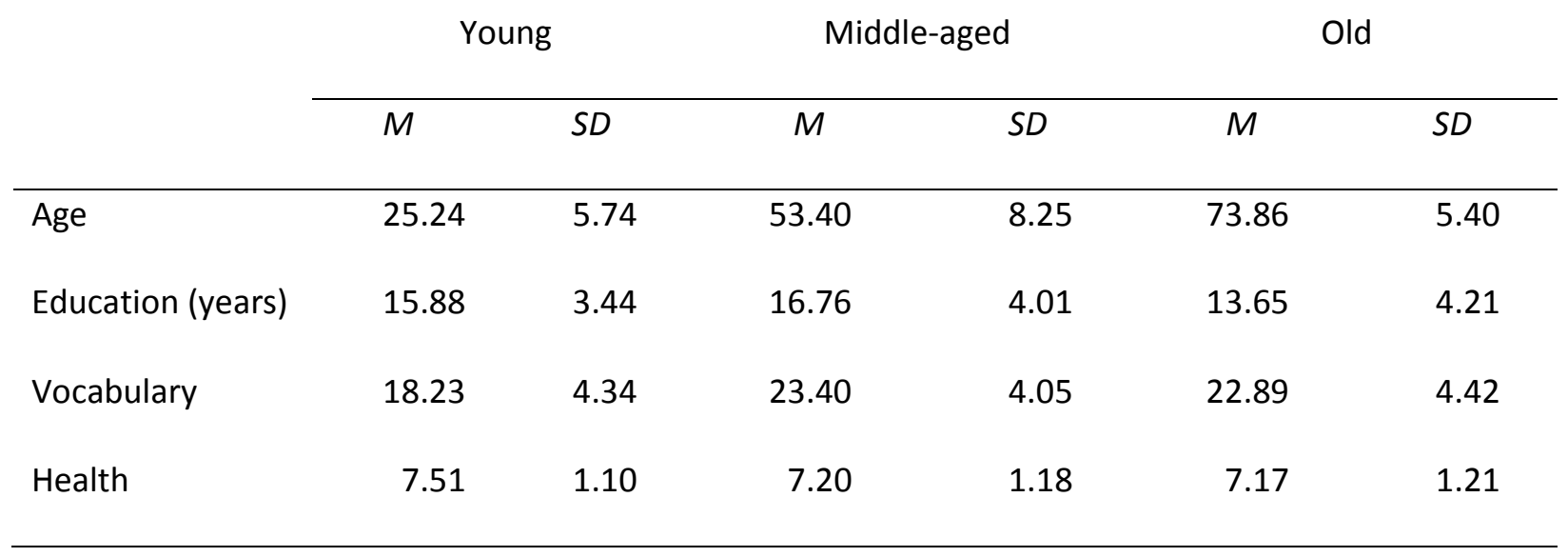


Table 2:

Descriptive statistics for age group differences in potential mediator variables and correlations with sarcasm tasks.

\begin{tabular}{lcccccccc}
\hline & \multicolumn{2}{c}{ Young } & \multicolumn{2}{c}{ Middle-aged } & \multicolumn{2}{c}{ Old } & \multicolumn{2}{c}{$\begin{array}{c}\text { Correlation with } \\
\text { sarcasm task: }\end{array}$} \\
\hline & $M$ & $S D$ & $M$ & $S D$ & $M$ & $S D$ & Video & Story \\
EP & 79.17 & 9.46 & 80.85 & 7.01 & 73.28 & 9.30 & $.379 * *$ & $.185 *$ \\
ToM & 73.71 & 11.67 & 75.72 & 8.58 & 70.73 & 9.99 & $.210 *$ & $.304 * *$ \\
2 2-back & 96.57 & 5.58 & 92.52 & 8.48 & 90.50 & 8.29 & .161 & $.188 \dagger$ \\
PANAS-N & 7.05 & 3.29 & 6.20 & 2.35 & 4.23 & 2.41 & .112 & .099 \\
PANAS-P & 13.91 & 4.74 & 10.63 & 4.12 & 14.10 & 6.45 & .073 & .011 \\
\hline
\end{tabular}

$\mathrm{EP}=$ emotion perception, $\mathrm{ToM}=$ theory of mind, PANAS-N and PANAS-P = negative affect and positive affect score on the Positive and Negative Affect Schedule. $† p=.057, * p<.05, * * p<.01$. 
Figure 1: Effects of age and video type on the percentage accuracy of understanding sincere, simple sarcastic and paradoxical sarcastic exchanges. Error bars are $1 \mathrm{SE}$ of the mean. $\mathrm{Y}=$ young, $\mathrm{M}=$ middleaged, $\mathrm{O}=$ old

Figure 2: Effects of age and story type $(\mathrm{MC}=$ mental control, $\mathrm{PC}=$ physical control, $\mathrm{DS}=$ direct sarcasm, IS = indirect sarcasm) on accuracy of understanding of story material. Errors bars are 1 SE of the mean. $Y$ = young, $\mathrm{M}=$ middle-aged, $\mathrm{O}=$ old.

Figure 3: Path diagrams of mediation analyses examining facial emotion perception (EP) as a possible mediator of age differences in sarcasm comprehension from (i) videos and (ii) stories. The numbers are uncorrected path coefficients as recommended in Preacher and Hayes (2008). The coefficients above the pathway between age and sarcasm comprehension $(c)$ are direct effects, while the bold figure in each case $\left(c^{\prime}\right)$ is the age effect on sarcasm comprehension once emotion perception is included as a mediator in the model. The significance of the mediation effect is determined by a bootstrapping procedure, see text for results. ${ }^{*} p<.05, * * p<.01, * * * p<.001$.

Figure 4: Path diagrams of mediation analyses examining working memory (WM) as a possible mediator of age differences in sarcasm comprehension from stories. The numbers are uncorrected path coefficients as recommended in Preacher and Hayes (2008). The coefficient above the pathway between age and sarcasm comprehension $(c)$ is the direct effect, while the bold figure $\left(c^{\prime}\right)$ is the age effect on sarcasm comprehension once WM is included as a mediator. Note that $\mathrm{n}=102$ for this analysis. ${ }^{*} p<$ $.05, * * p<.01, * * * p<.001$. 


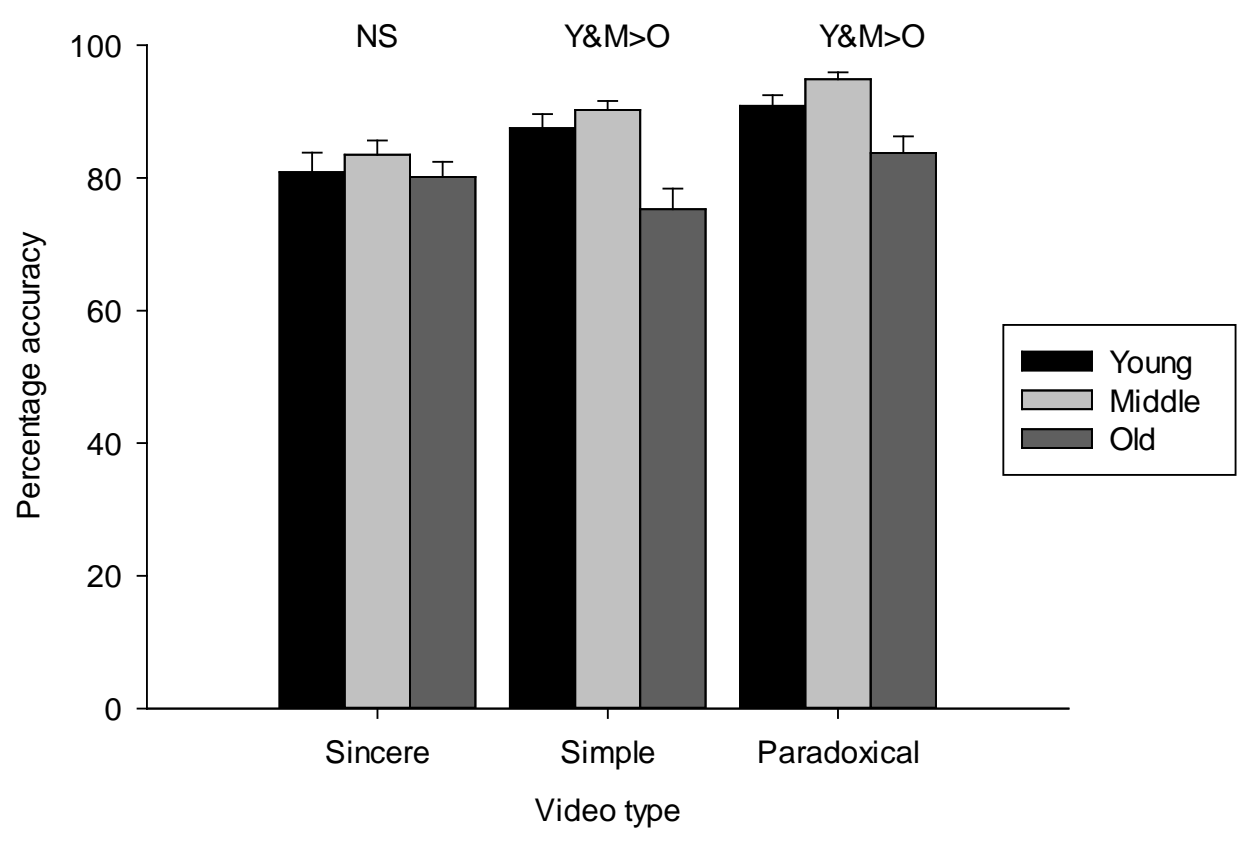




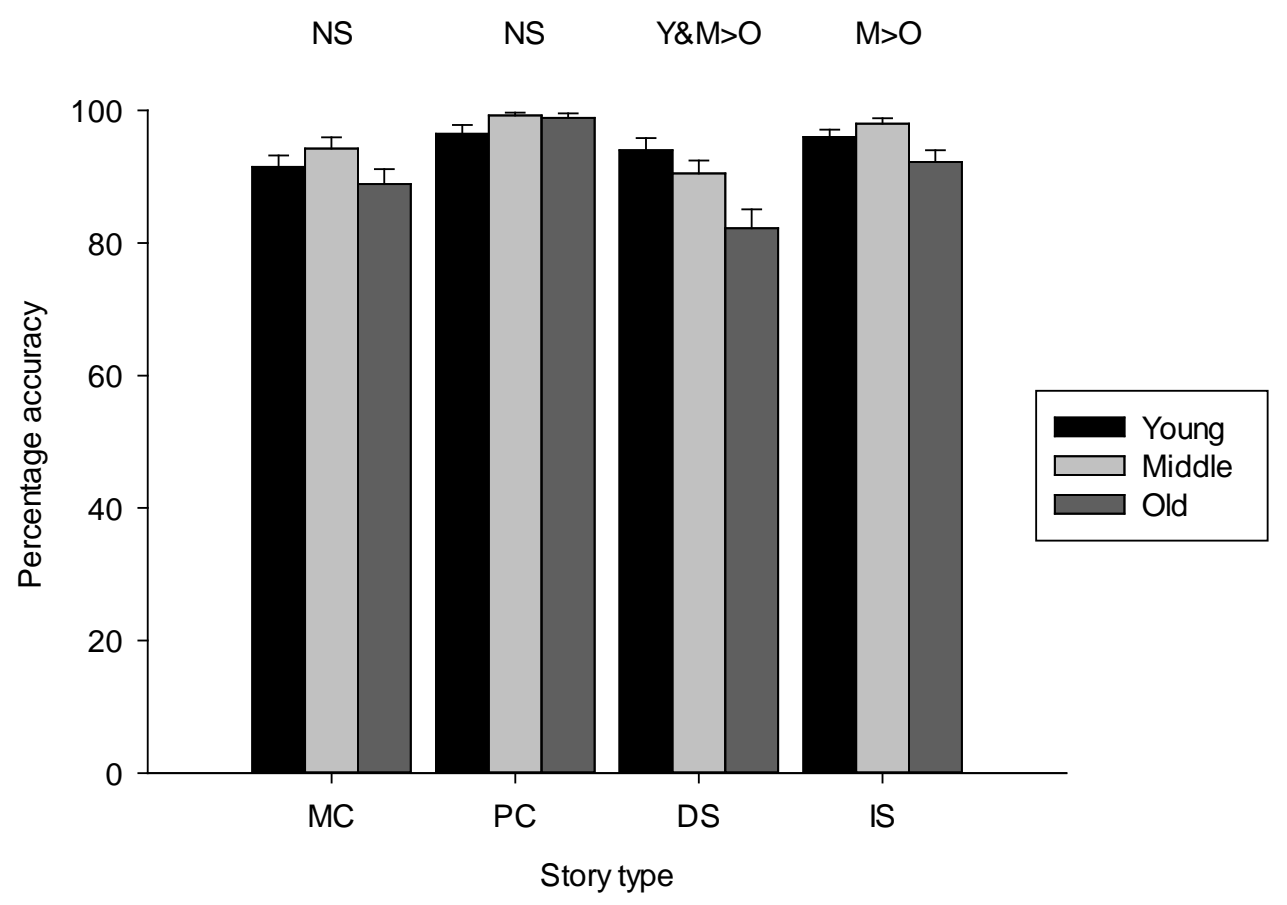


(i)

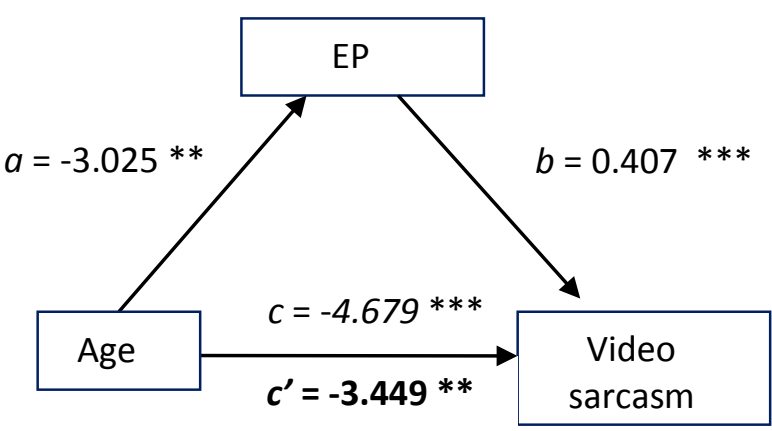

(ii)

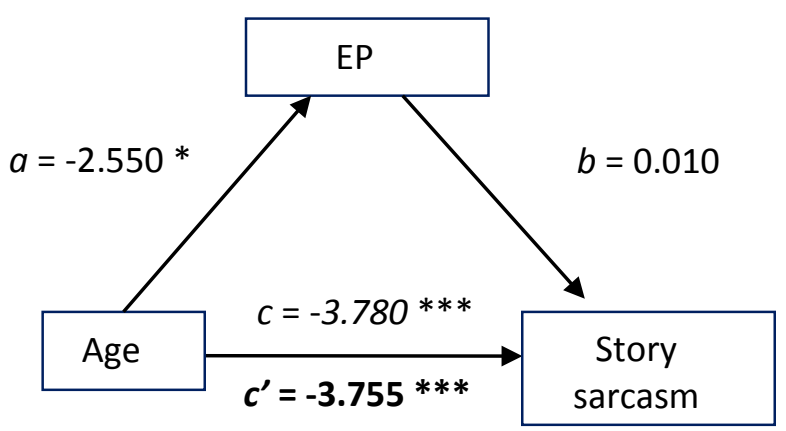




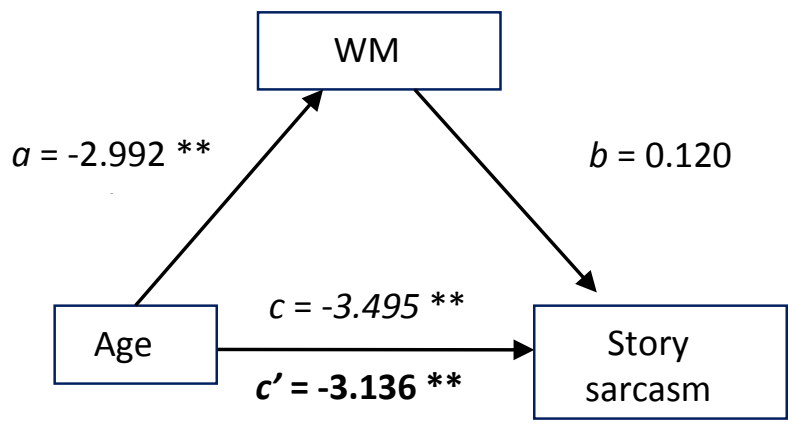


Appendix 1: Examples of stories from the sarcasm task.

\section{Example of physical control story}

Stephen's washing machine was broken. He hand-washed his black shirt and put it to dry on his balcony. It was summertime, and very hot and sunny. The shirt stayed on the balcony in the sunlight. Several days later he carried it indoors. The front was several shades lighter than the back. His mum said: "I did say you should have brought it in sooner!"

Why was the front lighter than the back?

1. It had been on the balcony.

2. The sunlight faded the colour on the front.

3. He had washed it by hand.

4. It had been raining.

\section{Example of mental control story}

Harriet thought she was overweight and was on a diet. She decided to go swimming early one morning. The pool was empty, and she started swimming. She swam one length of the pool. Suddenly, a group of teenage boys appeared. Harriet got out and left the swimming pool.

\section{Why did Harriet leave the pool?}

1. She thought it was too early in the morning.

2. She did not want to swim any longer.

3. She wanted everyone to look at her.

4. She did not want the boys to look at her.

\section{Example of direct sarcasm story}

Vicky had bought tickets for a new play at the theatre. One was for herself and the other for her friend Jean. Vicky said the play would be good. The play turned out to be terrible. They were both disappointed. Jean said: "That was a fantastic play you took me to see!"

\section{What did Jean mean when she said that?}

1. That play was terrible.

2. That was a very good play.

3. Next time l'll check what the critics say.

4. That was a beautiful theatre.

\section{Example of indirect sarcasm story}

One Wednesday evening Rob and his wife had a dinner party. Around midnight everyone was tired, and the guests started to leave. One man stayed and kept on talking. He finally left at four in the morning. He said that he hoped he had not stayed too long. Rob said: "We'll get at least two hours sleep before work tomorrow!"

What did the Rob mean when he said that?

1. We'll be able to sleep for two hours.

2. We won't get two hours sleep.

3. You did stay rather late.

4. I'll never invite you again. 